\title{
ANGULAR RIGHT SYMMETRICITY OF BOUNDED LINEAR OPERATORS ON HILBERT SPACES
}

\author{
Seyed Mohammad Sadegh Nabavi Sales \\ Hakim Sabzevari University, Iran
}

\begin{abstract}
We introduce and characterize angular right symmetric and approximate angular right symmetric points of the algebra of all bounded linear operators defined on either real or complex Hilbert spaces.
\end{abstract}

\section{INTRODUCTION}

This paper is a continuation of the works we have accomplished in $[11,12]$ in which we consider some definitions of angle and approximate orthogonality in normed spaces. In a real inner product space $(\mathscr{X},\langle\cdot, \cdot\rangle)$, the notion of angle between two non-zero vectors $\xi$ and $\eta$ is defined, very routinely. Indeed, $\xi{ }_{\theta} \eta$ for some $\theta \in[0, \pi]$ if and only if $\cos \theta=\frac{\langle\xi, \eta\rangle}{\|\xi\|\|\eta\|}$. In general real normed spaces in which norm does not necessarily come from an inner product, various types of definition were offered; see for example $[7,11,16]$. Especially, in [11] we introduce a definition for angle in real normed spaces related to the wellknown Birkhoff-James orthogonality. Recall that, for elements $\xi$ and $\eta$ in a complex or real normed space $(\mathscr{X},\|\cdot\|), \xi$ in the sense of Birkhoff-James is orthogonal to $\eta$, denoted by $\xi \perp_{B J} \eta$, if

$$
\|\xi+\lambda \eta\| \geq\|\xi\| \quad(\lambda \in \mathbb{K})
$$

which $\mathbb{K}$ stands for either, $\mathbb{C}$ or $\mathbb{R} ;[3,10]$. This type of orthogonality does not satisfy the property of symmetricity which means that there exist a normed space and vectors $\xi$ and $\eta$ in that normed space so that $\xi \perp_{B J} \eta$ but $\eta \not \perp_{B J} \xi$. In fact, it is well-known that symmetricity characterizes inner product spaces provided that the dimension of the space is greater than $2 ;[10]$.

2020 Mathematics Subject Classification. 47B49, 46C05, 46B20.

Key words and phrases. Angle in normed spaces, Birkhoff-James orthogonality, right symmetric points, angular right symmetric points. 
Given two vectors $\xi$ and $\eta$ in a real normed space, there exists a real number $\alpha$ so that $\xi \perp_{B J}(\alpha \xi+\eta)$ and $|\alpha| \leq \frac{\|\eta\|}{\|\xi\|}$. In general, there may exist a large number of such numbers. In fact, $\mathfrak{A}_{\xi, \eta}:=\left\{\alpha: \xi \perp_{B J}(\alpha \xi+\eta)\right\}$ is a nonempty closed interval. By $\mathfrak{A}_{\xi, \eta}>0$ (resp. $\mathfrak{A}_{\xi, \eta}<0$ ), we mean $\alpha>0$ (resp. $\alpha<0$ ) for all $\alpha \in \mathfrak{A}_{\xi, \eta}$.

Definition $1.1([11])$. Let $\xi, \eta \in \mathscr{X}$ and let

$$
\alpha_{0}= \begin{cases}\min \mathfrak{A}_{\xi, \eta}, & \mathfrak{A}_{\xi, \eta}>0 \\ \max \mathfrak{A}_{\xi, \eta}, & \mathfrak{A}_{\xi, \eta}<0 . \\ 0, & 0 \in \mathfrak{A}_{\xi, \eta}\end{cases}
$$

Define

$$
\cos (\xi, \eta)=\frac{-\alpha_{0}\|\xi\|}{\|\eta\|}
$$

We say that $\xi L_{\theta} \eta$, for some $\theta \in[0, \pi]$, if $\cos (\xi, \eta)=\cos \theta$. Also $\xi{ }_{\theta}^{\varepsilon} \eta$ for some $\varepsilon \geq 0$ and $\theta \in[0, \pi]$ whenever $|\cos (\xi, \eta)-\cos \theta| \leq \varepsilon$. In the case when $\theta=\frac{\pi}{2}$ this inequality introduces a type of approximate orthogonality with respect to the Bikhoff-James orthogonality which is the same as that introduced by Chmieliński in [5]; see [11]. Apparently, in these definitions we restrict ourselves to real normed spaces. However, in complex normed spaces we consider a generalized version of the definition: namely $\xi \angle_{\theta} \eta$ means $\xi \perp_{B J}$ $\left(\lambda \cos \theta \frac{\xi}{\|\xi\|}+\frac{\eta}{\|\eta\|}\right)$ for some $\lambda$ with $|\lambda|=1$. In [15] the author introduces the notions of right symmetric and left symmetric points in a real normed space. A vector $\xi$ in a normed space $\mathscr{X}$ (real or complex) is said to be left symmetric if $\xi \perp_{B J} \eta$ always implies $\eta \perp_{B J} \xi$, and is said to be right symmetric whenever $\eta \perp_{B J} \xi$ always implies $\xi \perp_{B J} \eta$. Particular attention is paid to these notions on the algebra of bounded linear operators; see [2, $8,13,17,18,19]$ and references therein. In this paper, we consider angular left and angular right symmetric points as follows: A vector $\xi$ in a normed space $\mathscr{X}$ is said to be angular left symmetric with respect to angle $\theta$, if $\xi{ }_{\theta} \eta$ always implies $\eta L_{\theta} \xi$, and is said to be angular right symmetric whenever $\eta \angle_{\theta} \xi$ always implies $\xi{ }_{\theta} \eta$. We also could consider approximate angular right symmetric and approximate angular left symmetric points. A vector $\xi$ is called an approximate right symmetric point if there exists $\varepsilon \in[0,1)$ so that $\eta \perp \xi$ always implies $\xi \perp_{B J}^{\varepsilon} \eta$ and is called an approximate left symmetric point if there exists $\varepsilon \in[0,1)$ so that $\xi \perp_{B J} \eta$ always implies $\eta \perp_{B J}^{\varepsilon} \xi$. The notions of approximate angular right and left symmetric points are defined in an obvious way. In this paper, we characterize angular right symmetric and approximate angular right symmetric points of the algebra of bounded operators defined on either real or complex Hilbert spaces. We, in fact, generalize the results of $[18]$ and $[8,14]$. We focus more closely on the issue of angular right symmetric points. The angular left symmetricity is somewhat different in two cases of 
complex and real. The problem we have considered in this paper discusses only operators defined on Hilbert spaces. We have to state that the problem of symmetricity of Birkhoff-James orthogonality for both cases of left and right for operators defined on either real or complex Hilbert spaces is fully responded in [19]. There, it is shown that right symmetric points in $\mathbb{B}(\mathscr{H})$ are isometries or coisometries multiplied by positive constants, while the only left symmetric point in $\mathbb{B}(\mathscr{H})$ is 0 . The problem arises naturally for operators defined on Banach spaces. For the special case of real orthogonality this problem is investigated in [9].

Throughout this paper, $\mathbb{B}(\mathscr{H})$ denotes the algebra of all bounded linear operators on a (real or complex) Hilbert space $\mathscr{H}$ and $S_{\mathscr{H}}$ is its unit sphere and $\mathbb{K}(\mathscr{H})$ is the ideal of all compact operators. Given an operator $T \in \mathbb{B}(\mathscr{H})$, $M_{T}$ is the set of norm attainment of $T$ consisting of all unit vectors $\xi$ for which $\|T \xi\|=\|T\|$. This set is nonempty whenever $\mathscr{H}$ is finite dimensional or $T \in \mathbb{K}(\mathscr{H})$. Let $\varepsilon>0$. By $B_{\varepsilon}(x)$ we mean the $\varepsilon$-neighborhood of $x$.

\section{Symmetricity of operators on COMPlex HilberT SPACES}

In this section, we investigate angular right symmetricity and approximation angular right symmetricity for operators which are defined on complex Hilbert spaces. For this study the following characterization of angle in $\mathbb{B}(\mathscr{H})$ is very essential. Its proof is done in light of [4, Theorem 1.1, Remark 3.1].

Theorem 2.1. Let $A, B \in \mathbb{B}(\mathscr{H})$ be non-zero and let $\theta \in[0, \pi]$. Then $A \angle{ }_{\theta} B$ if and only if there exists a sequence $\left\{\xi_{n}\right\}$ of unit vectors so that $\left\|A \xi_{n}\right\| \rightarrow\|A\|$ together with $\left\langle A \xi_{n}, B \xi_{n}\right\rangle \rightarrow \lambda \cos \theta\|A\|\|B\|$ for some $\lambda$ with $|\lambda|=1$. In addition, if $\mathscr{H}$ is finite dimensional, then the sequence $\left\{\xi_{n}\right\}$ can be replaced by $\xi \in \mathscr{H}$.

Proof. In the case when $\mathscr{H}$ is not finite dimensional $A \angle_{\theta} B$ means that there exists a $\lambda$ with $|\lambda|=1$ so that

$$
A \perp_{B J}\left(\lambda \cos \theta \frac{A}{\|A\|}+\frac{B}{\|B\|}\right)
$$

which by $\left[4\right.$, Remark 3.1] is equivalent to that there exists a sequence $\left\{\xi_{n}\right\}$ of unit vectors so that $\left\|A \xi_{n}\right\| \rightarrow\|A\|$ together with

$$
\left\langle A \xi_{n}, \lambda \cos \theta \frac{A}{\|A\|} \xi_{n}+\frac{B}{\|B\|} \xi_{n}\right\rangle \rightarrow 0 .
$$

Thus $\left\langle A \xi_{n}, B \xi_{n}\right\rangle \rightarrow-\bar{\lambda} \cos \theta\|A\|\|B\|$. In the finite dimensional case the proof is done by invoking [4, Theorem 1.1].

It is concluded immediately from the theorem that $1{ }_{\theta} A$ for some operator $A$, if and only if there exists $\lambda$ with $|\lambda|=1$ so that $\lambda \cos \theta \in \overline{W(A)}$ where $W(A)$ stands for the numerical range of an operator $A$ and $\overline{W(A)}$ is its closure.

We need the following lemmas. 
Lemma $2.2([18])$. Let $A \in \mathbb{B}(\mathscr{H})$ be positive and let $\|A\|=1$. If $\left\{\xi_{n}\right\}$ is a sequence of unit vectors such that $\left\|A \xi_{n}\right\| \rightarrow 1$, then $A \xi_{n}-\xi_{n} \rightarrow 0$.

The next two lemmas generalize [18, Lemma 2.3, Lemma 2.4]. Modifying the associated proofs, we prove these lemmas.

LEMma 2.3. Let $\theta \in\left(0, \frac{\pi}{2}\right]$ and $0<\theta_{1}<\theta$, and let

$$
A=\left(\begin{array}{cc}
\cos \theta_{1} & \sin \theta_{1} \\
\sin \theta_{1} & -\cos \theta_{1}
\end{array}\right) \quad \text { and } \quad B=\left(\begin{array}{cc}
1 & 0 \\
0 & m
\end{array}\right)
$$

where $m \in[0,1)$. Then the following holds.

(i) The numerical range $W(A B)$ is an ellipse and $\cos \theta$ is an interior point.

(ii) $A \angle_{\theta} B$ but not $B \angle_{\theta} A$.

Proof. (i) The matrix $A B$ is not normal so $W(A B)$ is an ellipse whose foci are the eigenvalues

$$
\begin{aligned}
\lambda(m) & =\frac{\cos \theta_{1}(1-m)+\sqrt{\cos ^{2} \theta_{1}(1-m)^{2}+4 m}}{2}, \\
\lambda^{\prime}(m) & =\frac{\cos \theta_{1}(1-m)-\sqrt{\cos ^{2} \theta_{1}(1-m)^{2}+4 m}}{2} .
\end{aligned}
$$

Obviously, $\lambda^{\prime}(m) \leq 0$ for all $m$. On the other hand, it is readily seen that

$$
\frac{\mathrm{d} \lambda(m)}{\mathrm{d} m}=-\frac{\cos \theta_{1}}{2}+\frac{4-2 \cos ^{2} \theta_{1}(1-m)}{2 \sqrt{\cos ^{2} \theta_{1}(1-m)^{2}+4 m}}>0
$$

hence, $\lambda(m)$ is an increasing function with respect to the parameter $m$ so its minimum takes place at $m=0$ which is $\lambda(0)=\cos \theta_{1}$. Hence,

$$
\cos \theta \in\left[0, \cos \theta_{1}\right] \subseteq\left[\lambda^{\prime}(m), \lambda(m)\right],
$$

for any $m \in[0,1)$. Therefore, $\cos \theta$ is an interior point of $W(A B)$.

(ii) Its proof is exactly the same as proof of (ii) in [18, Lemma 2.3] and for the sake of convenience we state it. Note that $\cos \theta \in W(A B)$, thus, $1 \angle_{\theta} A B$ which means

$$
1 \perp_{B J}\left(\lambda \cos \theta+\frac{A B}{\|A B\|}\right)
$$

for some $\lambda$ with $|\lambda|=1$. Obviously, $\|A B\|=1$ which implies that $1 \perp_{B J}$ $(\lambda \cos \theta+A B)$ that is

$$
\|1+\mu(\lambda \cos \theta+A B)\| \geq 1, \quad \mu \in \mathbb{C} .
$$

Now, we note that $A$ is a symmetry operator that is a self-adjoint unitary, hence,

$$
\left\|A+\mu\left(\lambda \cos \theta_{1} A+B\right)\right\| \geq\|A\|, \quad \mu \in \mathbb{C} .
$$


Thus, $A \angle_{\theta} B$. To show that $B \angle_{\theta} A$ does not hold we note that matrix $B$ attains its norm on unit vectors of the form $\xi=\left(\mathrm{e}^{\mathrm{i} \phi}, 0\right)$. Thus, using Theorem 2.1 we have

$$
\langle A \xi, B \xi\rangle=\cos \theta_{1}>\cos \theta,
$$

which completes the proof.

Note that $\cos \theta_{1}$ that appeared in the proof of this theorem is also an interior point of $W(A B)$. An extension of this lemma for the infinite dimensional case is stated below. This lemma, in fact, generalizes [18, Lemma 2.4].

Lemma 2.4. Let $B$ be positive, $\|B\|=1$ and $B \neq 1$ and let $\theta \in\left(0, \frac{\pi}{2}\right]$. Then, there exists an operator $A$ in $\mathbb{B}(\mathscr{H})$ such that $A \angle_{\theta} B$ but not $B \angle_{\theta} A$.

Proof. Let $m=\min \sigma(B)$. Hence, $0 \leq m<1$. Pick $0<\theta_{1}<\theta$ and let

$$
0<\varepsilon<\frac{1}{2} \min \left\{1-m, \operatorname{dist}\left(\cos \theta_{1}, \partial W\right), \operatorname{dist}(\cos \theta, \partial W), 1-\frac{\cos \theta}{\cos \theta_{1}}\right\}
$$

where $W(\cdot)$ is what appeared in the previous lemma and $\partial W(\cdot)$ stands for its boundary. Define function $f$ to be

$$
f(t)= \begin{cases}m, & m \leq t<m+\varepsilon \\ t, & m+\varepsilon \leq t \leq 1-\varepsilon \\ 1, & 1-\varepsilon<t \leq 1\end{cases}
$$

Set $C=f(B)$ and let $\mathscr{H}_{1}=E((1-\varepsilon, 1])(\mathscr{H}), \mathscr{H}_{2}=E([m, m+\varepsilon))(\mathscr{H})$ and $\mathscr{H}_{3}=E([m+\varepsilon, 1-\varepsilon])(\mathscr{H})$, where $E(\cdot)$ is the spectral measure associated to operator $B$. Thus, $\mathscr{H}=\mathscr{H}_{1} \oplus \mathscr{H}_{2} \oplus \mathscr{H}_{3}$, and with respect to this decomposition we may write

$$
B=B_{1} \oplus B_{2} \oplus B_{3} \quad \text { and } \quad C=1 \oplus m 1 \oplus B_{3} .
$$

Now, pick unit vectors $\xi \in \mathscr{H}_{1}$ and $\eta \in \mathscr{H}_{2}$ and define an operator $A$ on these three subspaces to be

$$
\begin{aligned}
& \mathscr{H}_{1} \quad: \quad A \xi=\cos \theta_{1} \xi+\sin \theta_{1} \eta,\left.\quad A\right|_{\xi^{\perp}}=1 \\
& \mathscr{H}_{2}: A \eta=\sin \theta_{1} \xi-\cos \theta_{1} \eta,\left.\quad A\right|_{\eta^{\perp}}=m 1 \\
& \mathscr{H}_{3}:\left.\right|_{\mathscr{H}_{3}}=B_{3},
\end{aligned}
$$

where orthogonal complement is considered with respect to the given subspace. Note that $\|A\|=1$. Here, we want to show that $A \angle_{\theta} B$. Let $P$ be the orthogonal projection on the two dimensional subspace spanned by $\xi$ and $\eta$. Thus,

$$
P A P=\left(\begin{array}{rr}
\cos \theta_{1} & \sin \theta_{1} \\
\sin \theta_{1} & -\cos \theta_{1}
\end{array}\right) \quad \text { and } \quad P C P=\left(\begin{array}{rr}
1 & 0 \\
0 & m
\end{array}\right)
$$


with respect to the basis $\{\xi, \eta\}$. So $W=W((P A P)(P C P))$ is an ellipse in the previous lemma and $\cos \theta_{1}$ and $\cos \theta$ are both interior points of this ellipse. Now, we note that $1-\varepsilon \leq\|P B P\| \leq 1$ which implies that

$$
\cos \theta \leq \frac{\cos \theta}{\|P B P\|} \leq \frac{\cos \theta}{1-\varepsilon}<\cos \theta_{1}
$$

where the last inequality is valid according to our assumption in the choice of $\varepsilon$. From the estimate

$|\langle(P A P)(P B P)-(P A P)(P C P) \zeta, \zeta\rangle| \leq\|P A P\|\|P B P-P C P\| \leq\|B-C\| \leq \varepsilon$

for all $\|\zeta\|=1$, and the choice of $\varepsilon$, it follows that $W((P A P)(P B P))$ is an ellipse and $\cos \theta_{1}$ and $\cos \theta$ are interior points of that. Hence, (2.1) implies that $\frac{\cos \theta}{\|P B P\|}$ is an interior point of $W((P A P)(P B P))$. Therefore,

$$
P A P \perp_{B J}\left(\frac{\cos \theta}{\|P B P\|} P A P+\frac{P B P}{\|P B P\|}\right)
$$

which implies

$$
P A P \perp_{B J}(\cos \theta P A P+P B P) .
$$

Thus,

$1=\|A\|=\|P A P\| \leq\|P A P+\gamma(\cos \theta P A P+P B P)\| \leq\|A+\gamma(\cos \theta A+B)\|$

for all $\gamma \in \mathbb{C}$ which means $A \angle_{\theta} B$.

The assertion that $B \angle_{\theta} A$ does not hold is done by the same method as that of [18, Lemma 2.4] however we provide a proof. Firstly, we need the following fact. If $\zeta$ is a unit vector in $\mathscr{H}_{1}$ then

$$
\langle\zeta, A \zeta\rangle \geq \cos \theta_{1} .
$$

To see this, write $\zeta=\lambda \xi+\mu \xi^{\perp}$ where $\xi^{\perp}$ is chosen to be in $\mathscr{H}_{1}$ with $\xi \perp \xi^{\perp}$ and $\lambda, \mu \in \mathbb{C}$. Therefore,

$$
\begin{aligned}
\langle\zeta, A \zeta\rangle & =\left\langle\lambda \xi+\mu \xi^{\perp}, \lambda\left(\cos \theta_{1} \xi+\sin \theta_{1} \eta\right)+\mu \xi^{\perp}\right\rangle=|\lambda|^{2} \cos \theta_{1}+|\mu|^{2} \\
& =|\lambda|^{2} \cos \theta_{1}+1-|\lambda|^{2}=1-\left(1-\cos \theta_{1}\right)|\lambda|^{2} \geq \cos \theta_{1} .
\end{aligned}
$$

Now, suppose that $\left\{\xi_{n}\right\}$ is a sequence of unit vectors such that $\left\|B \xi_{n}\right\| \rightarrow 1$. Then, by Lemma 2.2 we have that $B \xi_{n}-\xi_{n} \rightarrow 0$. We could write $\xi_{n}=$ $\sum_{i=1}^{3} \xi_{i, n}$ where $\xi_{i, n} \in \mathscr{H}_{i}$ for $i \in\{1,2,3\}$ and $n \in \mathbb{N}$ and $\sum_{i=1}^{3}\left\|\xi_{i, n}\right\|^{2}=1$. Then $B \xi_{n}-\xi_{n} \rightarrow 0$ implies $B \xi_{i, n}-\xi_{i, n} \rightarrow 0$. Now, we want to show that $\left\|\xi_{i, n}\right\| \rightarrow 0$ for $i=2,3$. Assume $\left\|\xi_{2, n}\right\| \rightarrow a$ for some $a \neq 0$. Thus, there exists $N \in \mathbb{N}$ so that $\left\{\frac{1}{\left\|\xi_{2, n}\right\|}\right\}_{n=N}^{\infty}$ is bounded. Therefore,

$$
\left\{\frac{1}{\left\|\xi_{2, n}\right\|}\left(B \xi_{2, n}-\xi_{2, n}\right)\right\}_{n=N}^{\infty}
$$


converges to 0 . Hence, $\left\|B_{2}\right\| \geq 1$ which is impossible. It follows that $\xi_{2, n} \rightarrow 0$. Similarly, we could show that $\xi_{3, n} \rightarrow 0$. Therefore,

$$
\left\langle B \xi_{n}, A \xi_{n}\right\rangle \rightarrow\left\langle\xi_{1, n}, A \xi_{1, n}\right\rangle \geq \cos \theta_{1}>\cos \theta
$$

which implies that $\left\langle B \xi_{n}, A \xi_{n}\right\rangle \not \rightarrow \lambda \cos \theta$ for any modulus one $\lambda \in \mathbb{C}$. Thus, $B{ }_{\theta} A$ cannot occur and we are done.

Now, we can extend [18, Theorem 2.5] in which the author gives a characterization of isometries and coisometries via right symmetricity of BJ-orthogonality. We may generalize this result by giving a characterization of isometries and coisometries through the angular right symmetricity and approximation angular right symmetricity as follows. In its proof we use the result of $[19$, Lemma 3.1] which states that for an operator $T \in \mathbb{B}(\mathscr{H})$, either $U$ in the polar decomposition $T=U|T|$ or $V$ in the polar decomposition $T^{*}=V\left|T^{*}\right|$ can be chosen to be isometry.

THEOREM 2.5. Let $B \in \mathbb{B}(\mathscr{H})$. The following statements are equivalent:

(i) $B$ is a scalar multiple of an isometry or coisometry,

(ii) $A \perp_{B J} B \Rightarrow B \perp_{B J} A$ for all $A \in \mathbb{B}(\mathscr{H})$,

(iii) $A \perp_{B J} B \Rightarrow B \perp_{B J}^{\varepsilon} A$ for all $A \in \mathbb{B}(\mathscr{H})$ and $\varepsilon \in[0,1)$,

(iv) there exist $\theta \in\left(0, \frac{\pi}{2}\right]$ and $\varepsilon \in[0,1-\cos \theta)$ so that $A \angle_{\theta} B \Rightarrow B \angle_{\theta}^{\varepsilon} A$ for all $A \in \mathbb{B}(\mathscr{H})$.

Proof. (i) $\Rightarrow$ (ii) is proved in $[18$, Theorem 2.5] and (ii) $\Rightarrow$ (iii) is obvious. For (iii) $\Rightarrow$ (i) choose $\theta_{0} \in\left(0, \frac{\pi}{2}\right]$ so that $\varepsilon<\cos \theta_{0}<1$ and assume that $\|B\|=1$ and $U$ is isometry in the polar decomposition $B=U|B|$ but $|B| \neq 1$. Thus, according to Lemma 2.4 there exists $A \in \mathbb{B}(\mathscr{H})$ so that $A \perp_{B J}|B|$ and $|B| \angle_{\theta_{1}} A$ for some $0 \leq \theta_{1} \leq \theta_{0}$ such that for $\theta_{1}<\theta$ the relation $|B| \angle_{\theta_{1}} A$ does not hold. Hence, $A \perp_{B J}|B|$ but $|B| \not \perp_{B J}^{\varepsilon} A$. Since $U$ is an isometry, we have that $U A \perp_{B J} B$ but $B \not \perp_{B J}^{\varepsilon} U A$ that contradicts the assumption. Therefore, $|B|=1$. If $V$ is an isometry in the polar decomposition $B^{*}=V\left|B^{*}\right|$, then similarly we could show that $B^{*}$ is an isometry.

(i) $\Rightarrow$ (iv) Assume that $\|B\|=1$. If $B$ is an isometry and $A \angle_{\theta} B$ then, by Theorem 2.1, there exists a sequence of unit vectors $\left\{\xi_{n}\right\}$ so that $\left\|A \xi_{n}\right\| \rightarrow$ $\|A\|$ and $\left\langle A \xi_{n}, B \xi_{n}\right\rangle \rightarrow \lambda \cos \theta$ for some modulus one $\lambda \in \mathbb{C}$. But, then $\left\langle B \xi_{n}, A \xi_{n}\right\rangle \rightarrow \bar{\lambda} \cos \theta$ and $\left\|B \xi_{n}\right\|=\left\|\xi_{n}\right\|=1=\|B\|$ because $B$ is an isometry which implies that $B{ }_{\theta} A$ according to Theorem 2.1 and obviously this implies that $B \angle_{\theta}^{\varepsilon} A$. In the case when $B^{*}$ is an isometry the proof is accomplished by the same method because $A \angle_{\theta} B$ if and only if $A^{*} \angle_{\theta} B^{*}$.

(iv) $\Rightarrow$ (i) Let $B=U|B|$ be the polar decomposition of $B, U$ be an isometry, and let $\|B\|=1$. Assume that $B$ is not an isometry, that means $B^{*} B \neq 1$, and pick $\theta_{0} \in\left(0, \frac{\pi}{2}\right]$ so that $\cos \theta+\varepsilon<\cos \theta_{0}$. Therefore, according to Lemma 2.4 , there exists an operator $A \in \mathbb{B}(\mathscr{H})$ with $\|A\|=1$ so that $A \angle_{\theta}|B|$ and $|B| \angle_{\theta_{1}} A$ for some $\theta_{1} \leq \theta_{0}$ but $|B| \angle_{\eta} A$ does not hold for any $\theta_{1}<\eta$. Therefore, 
$A \angle_{\theta}|B|$ but not $|B| \angle_{\theta}^{\varepsilon} A$ and since $U$ is an isometry $U A \angle_{\theta} B$ but not $B \angle_{\theta}^{\varepsilon} U A$ which contradicts (iv). Thus, $|B|=1$, i.e., $B$ is an isometry.

The proof of the following theorem is done by a similar method.

Theorem 2.6. Let $B \in \mathbb{B}(\mathscr{H})$ and let $0<\theta_{2} \leq \theta_{1} \leq \frac{\pi}{2}$. If $A \angle_{\theta_{1}} B \Rightarrow$ $B \angle_{\theta_{2}} A$ for all $A \in \mathbb{B}(\mathscr{H})$, then $B$ is a scalar multiple of an isometry or coisometry.

\section{Symmetricity of operators on Real Hilbert spaces}

At this point, we pay attention to operators which are defined on real Hilbert spaces satisfying angular right symmetricity and approximation angular right symmetricity. The method of this section is independent of what utilized in the previous section and it refers mainly to that used in [8]. In [8] the authors prove that for a linear operator $T$ defined on a real finite dimensional Hilbert space $\mathscr{H}, A \perp_{B J} T \Rightarrow T \perp_{B J} A$ for all $A \in \mathbb{B}(\mathscr{H})$ if and only if $M_{T}=S_{\mathscr{H}}$ which implies that $T$ is a unitary multiplied by a positive constant. In fact, they show that if $M_{T} \neq S_{\mathscr{H}}$ and $\|T\|=1$, then there exists an operator $A$ with $\|A\|=1$ so that $A \perp_{B J} B$ but $T \| A$. In this case, an easy calculation, following their method, shows that for such $A$ and $T$ and for any $\theta \in\left[0, \frac{\pi}{2}\right]$ we have that $A \perp_{B J}(\cos \theta A+T)$. To see this, according to [8], let $M_{T}=S_{\mathscr{H}_{0}}$ for some subspace $\mathscr{H}_{0}$ of $\mathscr{H}$ and assume that $\left\{\xi_{1}, \ldots, \xi_{m}, \ldots, \xi_{n}\right\}$ is an orthonormal basis for $\mathscr{H}$ such that $\left\{\xi_{1}, \ldots, \xi_{m}\right\}$ forms an orthonormal basis for $\mathscr{H}_{0}$, and choose a unit vector $w_{0} \in T\left(\mathscr{H}_{0}\right)^{\perp}$. Now, suppose that $\left\|w_{0}+\lambda\left(\cos \theta w_{0}+T \xi_{m+1}\right)\right\| \geq 1$ for any $\lambda \geq 0$. In this case, $A$ is defined to be $A \xi_{i}=-T \xi_{i}$ for $i \in\{1, \ldots, m\}, A \xi_{m+1}=w_{0}$, and $A \xi_{i}=0$ for $i \in\{m+2, \ldots, n\}$. Thus, for $\zeta=\sum_{i=1}^{n} c_{i} \xi_{i} \in S_{\mathscr{H}}$ we have that $\|A \zeta\|^{2}=\sum_{i=1}^{m} c_{i}^{2}+c_{m+1}^{2} \leq 1$ which by $A \xi_{m+1}=w_{0}$ implies that $\|A\|=1$. Hence, for $\lambda>0$,

$$
\begin{aligned}
\|A+\lambda(\cos \theta A+T)\| & \geq\left\|A \xi_{m+1}+\lambda\left(\cos \theta A \xi_{m+1}+T \xi_{m+1}\right)\right\| \\
& =\left\|w_{0}+\lambda\left(\cos \theta w_{0}+T \xi_{m+1}\right)\right\| \geq 1=\|A\|
\end{aligned}
$$

and for $\lambda<0$ we have

$$
\begin{aligned}
\|A+\lambda(\cos \theta A+T)\| & \geq\left\|A \xi_{m}+\lambda\left(\cos \theta A \xi_{m}+T \xi_{m}\right)\right\| \\
& =\left\|-T \xi_{m}-\lambda \cos \theta T \xi_{m}+\lambda T \xi_{m}\right\| \\
& =|-1-\lambda \cos \theta+\lambda|\|T\| \\
& =|1-\lambda(1-\cos \theta)| \\
& \geq 1=\|A\|,
\end{aligned}
$$

i.e., $\cos \theta \in \mathfrak{A}_{(A, T)}$. If $\left\|w_{0}+\lambda\left(\cos \theta w_{0}+T \xi_{m+1}\right)\right\| \geq 1$ for any $\lambda \leq 0$, then $A$ is defined to be $A \xi_{i}=T \xi_{i}$ for $i \in\{1, \ldots, m\}, A \xi_{m+1}=w_{0}$, and $A \xi_{i}=0$ for $i \in\{m+2, \ldots, n\}$ and the rest is the same as above. Observing these facts, we could improve [8, Theorem 2.7]. 
TheOREm 3.1. Let $\mathscr{H}$ be a finite dimensional real Hilbert space and $T \in$ $\mathbb{B}(\mathscr{H})$. The following statements are equivalent:

(i) $M_{T}=S_{\mathscr{H}}$,

(ii) $A \perp_{B J} T \Rightarrow T \perp_{B J} A$ for all $A \in \mathbb{B}(\mathscr{H})$,

(iii) there exists an $\varepsilon \in[0,1)$ so that $A \perp_{B J} T \Rightarrow T \perp_{B J}^{\varepsilon} A$ for any $A \in \mathbb{B}(\mathscr{H})$,

(iv) there exist $a \theta \in\left(0, \frac{\pi}{2}\right]$ and $a 0 \leq \varepsilon<1-\cos \theta$ so that $\cos \theta \in$ $\mathfrak{A}_{\left(\frac{A}{\|A\|}, \frac{T}{\|T\|}\right)} \Rightarrow B_{\varepsilon}(\cos \theta) \cap \mathfrak{A}_{\left(\frac{T}{\|T\|}, \frac{A}{\|A\|}\right)} \neq \emptyset$ for any $A \in \mathbb{B}(\mathscr{H})$.

Proof. Implications (i) $\Rightarrow($ ii $) \Rightarrow$ (iii) are obvious. For (iii) $\Rightarrow($ i) assume that $M_{T} \neq S_{\mathscr{H}}$ and $\|T\|=1$. Thus, there exists an operator $A$ with $\|A\|=1$ so that $A \perp_{B J} T$ but $T \| A$ that implies $T \perp_{B J} T+A$ but $T \not \perp_{B J} \lambda \varepsilon T+A$ for $0 \leq \varepsilon<1$ and $-1 \leq \lambda \leq 1$ which contradicts (iii). For (i) $\Rightarrow$ (iv) assume that $\cos \theta \in \mathfrak{A}_{\left(\frac{A}{\|A\|}, \frac{T}{T T}\right)}$ thus, there exists $\xi_{0} \in S_{\mathscr{H}}$ so that $\left\|A \xi_{0}\right\|=\|A\|$ and $\left\langle A \xi_{0}, T \xi_{0}\right\rangle=\lambda \cos \theta\|A\|\|T\|$ which implies that $\left\langle T \xi_{0}, A \xi_{0}\right\rangle=\lambda \cos \theta\|A\|\|T\|$ and $\left\|T \xi_{0}\right\|=\|T\|$, because $M_{T}=S_{\mathscr{H}}$. Thus $\cos \theta \in B_{\varepsilon}(\cos \theta) \cap \mathfrak{A}_{\left(\frac{T}{\|T\|}, \frac{A}{\|A\|}\right)}$. For the converse assume that $M_{T} \neq S_{\mathscr{H}}$ and $\|T\|=1$. Therefore, there exists an operator $A$ with $\|A\|=1$ so that $A \perp_{B J} \cos \theta A+T$ and $T \| A$. Hence, $\cos \theta \in \mathfrak{A}_{(A, T)}$ but $B_{\varepsilon}(\cos \theta) \cap \mathfrak{A}_{(T, A)}=\emptyset$.

The following result is proved similarly and we omit its proof.

TheOrem 3.2. Let $\mathscr{H}$ be a finite dimensional real Hilbert space and $T \in$ $\mathbb{B}(\mathscr{H})$ and let $\theta \in\left(0, \frac{\pi}{2}\right]$. If $T \perp_{B J} A$ always implies that $A \angle_{\theta} T$, then $M_{T}=$ $S_{\mathscr{H}}$.

In the case when operators act on an infinite dimensional Hilbert space $\mathscr{H}$, it is proved in [8] that an orthogonal right symmetric operator $T$ is 0 , whenever $T$ is compact. Here, we could generalize this result noticing some facts from [8]. In [8] the authors prove that if $T$ is a compact operator of norm one, then there exists a norm one operator $A$ such that $A \perp_{B J} T$ but $T \| A$. It is readily checked that for $\theta \in\left[0, \frac{\pi}{2}\right]$ for such operators $T$ and $A$ we have that $\cos \theta \in \mathfrak{A}_{(A, T)}$. Thus, using the same argument as that of Theorem 3.1 or $[8$, Theorem 2.8], we could prove the following two theorems.

THEOREM 3.3. Let $\mathscr{H}$ be an infinite dimensional real Hilbert space and $T \in \mathbb{K}(\mathscr{H})$. The following statements are equivalent:

(i) $T=0$,

(ii) $A \perp_{B J} T \Rightarrow T \perp_{B J} A$ for all $A \in \mathbb{B}(\mathscr{H})$,

(iii) there exists an $\varepsilon \in[0,1)$ so that $A \perp_{B J} T \Rightarrow T \perp_{B J}^{\varepsilon} A$ for any $A \in \mathbb{B}(\mathscr{H})$,

(iv) there exist $a \theta \in\left(0, \frac{\pi}{2}\right]$ and $a 0 \leq \varepsilon<\min \{\cos \theta, 1-\cos \theta\}$ so that $\cos \theta \in \mathfrak{A}_{\left(\frac{A}{\|A\|}, \frac{T}{\|T\|}\right)} \Rightarrow B_{\varepsilon}(\cos \theta) \cap \mathfrak{A}_{\left(\frac{T}{\|T\|}, \frac{A}{\|A\|}\right)} \neq \emptyset$ for all $A \in \mathbb{B}(\mathscr{H})$. 
TheOREM 3.4. Let $\mathscr{H}$ be an infinite dimensional real Hilbert space and $T \in \mathbb{K}(\mathscr{H})$ and let $\theta \in\left(0, \frac{\pi}{2}\right]$. If $T \perp_{B J} A$ always implies that $A \angle_{\theta} T$, then $T=0$.

The results we state about angular symmetricity do not say anything on the issue of parallelism. If we want to raise symmetricity of parallelism, what does the issue look like? More precisely:

Let $T$ be an operator so that $T \| A$ whenever $A \| T$. What can we say about $T$ in cases of real and complex? Is $T$ an isometry or coisometry multiplied by a constant? By Proposition 4.1 in [1] we see that when $T \| A$ then there exists a $\lambda \in \mathbb{C}$ with $|\lambda|=1$ so that $\lambda \in \mathfrak{A}_{\left(\frac{A}{\|A\|}, \frac{T}{\| T \pi}\right)}$.

Also, the results we establish for the angular symmetricity in the real case are proved under a limited condition of compactness. In [19] we do not see any such restriction on operators for the special case of symmetricity of orthogonality. Is it possible to generalize these results and achieve better conclusions stating some similar results for symmetricity of angle without such restrictions?

In [6, Definition 2.1] the authors introduce the notion of approximate symmetry of Birkhoff-James orthogonality in real normed spaces as follows. The Birkhoff-James orthogonality relation in a normed space $\mathscr{X}$ is called $\varepsilon$ symmetric for some $\varepsilon \in[0,1)$, if for any $x, y \in \mathscr{X}, x \perp_{B J} y$ always implies that $y \perp_{B J}^{\varepsilon} x$ (here we consider the definition for two cases of real and complex). Our results show that for all $\varepsilon \in[0,1)$ the Birkhoff-James orthogonality in $\mathbb{B}(\mathscr{H})$ is not $\varepsilon$-symmetric.

Finally, we present a characterization of isometries through the notion of norm attainment set of an operator defined between normed spaces. We use essentially Proposition 2.1 of [14]. It asserts that for an operator $T$ and vector $\xi \in M_{T}, T \xi \perp_{B J} T \eta$ always implies $\xi \perp_{B J} \eta$. Note that, this theorem is stated for operators defined between Banach spaces.

Theorem 3.5. Let T: $\mathscr{X} \rightarrow \mathscr{Y}$ be a bounded linear operator between real Banach spaces and let $\xi \in M_{T}$. Then $T$ is an isometry multiplied by a positive constant if and only if $T$ is injective and $T \xi{ }_{\theta} T \eta$ implies that $\xi \angle_{\theta} \eta$ for all $\eta \in \mathscr{X}$ and all $\theta \in[0, \pi]$.

Proof. First, assume that $T$ is an isometry multiplied by a positive number, $c$ say. Thus, $\|T \eta\|=c\|\eta\|$ for any $\eta \in \mathscr{X}$. Now, consider $\theta \in\left[0, \frac{\pi}{2}\right)$ and pick $0 \neq \eta \in \mathscr{X}$ with $T \xi \angle_{\theta} T \eta$. Since $T$ is injective, $T \xi \angle_{\theta} T \eta$ means that

$$
T \xi \perp_{B J}\left(\cos \theta \frac{T \xi}{\|T \xi\|}+\frac{T \eta}{\|T \eta\|}\right)
$$

and for $s<\cos \theta$,

$$
T \xi \not L_{B J}\left(s \frac{T \xi}{\|T \xi\|}+\frac{T \eta}{\|T \eta\|}\right) .
$$


Therefore, by Proposition 2.1 of [14] and the assumption we have that

$$
\xi \perp_{B J}\left(\cos \theta \frac{\xi}{\|T\|}+\frac{\eta}{\|T\|\|\eta\|}\right),
$$

that is,

$$
\xi \perp_{B J}\left(\cos \theta \xi+\frac{\eta}{\|\eta\|}\right) .
$$

Now, note that $\cos \theta>0$ and let $s$ be a number with $s<\cos \theta$ so that $\xi \perp_{B J}\left(s x+\frac{\eta}{\|\eta\|}\right)$. Since $\frac{T}{\|T\|}$ is an isometry we have that

$$
\frac{T \xi}{\|T\|} \perp_{B J}\left(s \frac{T \xi}{\|T\|}+\frac{T \eta}{\|T\|\|\eta\|}\right)
$$

that implies

$$
T \xi \perp_{B J}\left(s \frac{T \xi}{\|T \xi\|}+\frac{T \eta}{\|T \eta\|}\right),
$$

and this contradicts (3.1) thus, $\xi \angle_{\theta} \eta$. For the cases when $\cos \theta \leq 0$ the proof is similar.

For the converse assume that $\eta \in \mathscr{X}$. Thus, there exists a unique $\theta \in[0, \pi]$ so that $T \xi \angle_{\theta} T \eta$ which is

$$
T \xi \perp_{B J}\left(\cos \theta \frac{T \xi}{\|T \xi\|}+\frac{T \eta}{\|T \eta\|}\right)
$$

because $T$ is injective. Without loss of generality, we may assume that $\cos \theta>$ 0 . Therefore, $T \xi \not \not_{B J}\left(s \frac{T \xi}{\|T \xi\|}+\frac{T \eta}{\|T \eta\|}\right)$ for any $s<\cos \theta$. Hence, according to [14, Proposition 2.1], we have that

$$
\xi \perp_{B J}\left(\cos \theta \frac{\|T \eta\| \xi}{\|T\|\|\eta\|}+\frac{\eta}{\|\eta\|}\right) .
$$

On the other hand, equation (3.2) getting together with the assumption imply that $\xi \angle_{\theta} \eta$, that is, $\xi \perp_{B J}\left(\xi \cos \theta+\frac{\eta}{\|\eta\|}\right)$ but

$$
\xi \not \chi_{B J}\left(s \xi+\frac{\eta}{\|\eta\|}\right)
$$

for $s<\cos \theta$. If $\|T \eta\|<\|T\|\|\eta\|$, then $s_{0}=\cos \theta \frac{\|T \eta\|}{\|T\|\|\eta\|}<\cos \theta$ which by (3.3) means $\xi \perp_{B J}\left(s_{0} \xi+\frac{\eta}{\|\eta\|}\right)$ that contradicts (3.4), thus, $\|T \eta\|=\|T\|\|\eta\|$.

\section{ACKNOWLEDGEMENTS.}

The author would like to sincerely thank the referee for very careful reading of the paper and for their valuable comments improving the paper. 


\section{REFERENCES}

[1] Lj. Arambašić, R. Rajić, The Birkhoff-James orthogonality in Hilbert $C^{*}$-modules, Linear Algebra Appl. 437 (2012), 1913-1929.

[2] Lj. Arambašić, R. Rajić, On symmetry of the (strong) Birkhoff-James orthogonality in Hilbert $C^{*}$-modules, Ann. Funct. Anal. 7 (2016), 17-23.

[3] G. Birkhoff, Orthogonality in linear metric spaces, Duke Math. J. 1 (1935), 169-172.

[4] R. Bhatia and P. Šemrl, Orthogonality of matrices and some distance problems, Linear Algebra Appl. 287 (1999), 77-86.

[5] J. Chmieliński, On an $\varepsilon$-Birkhoff orthogonality, J. Inequal. Pure Appl. Math. 6 (2005), Art. 79,7 pp.

[6] J. Chmieliński and P. Wójcik, Approximate symmetry of Birkhoff orthogonality, J. Math. Anal. Appl. 461 (2018), 625-640.

[7] C. R. Diminnie, E. Z. Andalafte and R. W. Freese, Angles in normed linear spaces and a characterization of real inner product spaces, Math. Nachr. 129 (1986), 197-204.

[8] P. Ghosh, D. Sain and K. Paul, Orthogonality of bounded linear operators, Linear Algebra Appl. 500 (2016), 43-51.

[9] P. Ghosh, D. Sain and K. Paul, Symmetry of Birkhoff-James orthogonality of bounded linear operators, Adv. Oper. Theory 2 (2017), 428-434.

[10] R. C. James, Orthogonality and linear functionals in normed linear spaces, Trans. Amer. Math. Soc. 61 (1947), 265-292.

[11] S. M. S. Nabavi Sales, On mappings which approximately preserve angles, Aequationes Math. 92 (2018), 1079-1090.

[12] S. M. S. Nabavi Sales, On approximate Birkhoff-James orthogonality and approximate *-orthogonality in $C^{*}$-algebras, Sahand Communications in Mathematical Analysis 13 (2019), 153-163.

[13] K. Paul, A. Mal, P. Wójcik, Symmetry of Birkhoff-James orthogonality of operators defined between infinite dimensional Banach spaces, Linear Algebra. Appl. 563 (2019), 142-153.

[14] D. Sain, On norm attainment set of a bounded linear operator, J. Math. Anal. Appl. 457 (2018), 67-76.

[15] D. Sain, Birkhoff-James orthogonality of linear operators on finite dimensional Banach spaces, J. Math. Anal. Appl. 447 (2017), 860-866.

[16] T. Szostok, On a generalization of the sine function, Glas. Mat. Ser. III 38(58) (2003), 29-44.

[17] R. Tanaka and D. Sain, On symmetry of strong Birkhoff orthogonality in $B(H, K)$ and $K(H, K)$, Ann. Funct. Anal. 11 (2020), 693-704.

[18] A. Turnšek, On operators preserving James' orthogonality, Linear Algebra Appl. 407 (2005), 189-195.

[19] A. Turnšek, A remark on orthogonality and symmetry of operators in $\mathbb{B}(\mathscr{H})$, Linear Algebra Appl. 535 (2017), 141-150.

Department of Mathematics and Computer Sciences

Hakim Sabzevari University

P.O. Box 397, Sabzevar

Iran

E-mail: sadegh.nabavi@gmail.com \& sadegh.nabavi@hsu.ac.ir

Received: 15.4 .2020 .

Revised: 19.5.2020. 